\title{
Surgical Complications in Children with CDH: A Multivariate Analysis
}

\author{
Kim Heiwegen ${ }^{1}$ Iris A. L. M. van Rooij ${ }^{2}$ Arno van Heijst ${ }^{3}$ Ivo de Blaauw ${ }^{1}$. \\ Sanne M. B. I. Botden ${ }^{1}$
}

Published online: 31 January 2020

(C) The Author(s) 2020

\begin{abstract}
Introduction Several medical and surgical improvements in the treatment of congenital diaphragmatic hernia (CDH) patients have led to a higher survival rate. However, some of these improvements also lead to an increased morbidity rate. This study aims to determine the contribution different medical and surgical treatments have had on the development of surgical complications.

Method All CDH patients treated in a single centre between 2000 and 2015 were retrospectively evaluated. Multivariate logistic regression was used to estimate the independent effects of several treatment options that could influence the surgical outcome by adjustment for multiple risk factors.

Results Sixty of the 197 surgically repaired CDH patients had surgical complications. There were more haemorrhagic complications in the ECMO compared to non-ECMO group ( $27 \%$ vs. $2 \%, p<0.001)$. The use of inhaled nitric oxide was also significantly related to haemorrhage $(\mathrm{OR}=13.0(95 \%$ CI 1.1-159)). After adjustment for other risk factors, chylothorax was neither significantly associated with ECMO treatment $(\mathrm{OR}=1.6(95 \% \mathrm{CI}$ 0.5-5.2) nor with patch repair $(\mathrm{OR}=2.1: 95 \% \mathrm{CI}$ 0.7-6.1). A recurrence occurred more often in patients with pulmonary hypertension $(\mathrm{OR}=10.0(95 \% \mathrm{CI} 1.5-65.8)$ and after treatment with an abdominal patch $(\mathrm{OR}=11.3: 95 \% \mathrm{CI}$ 1.5-84.4).

Conclusion ECMO treatment and the inhalation of nitric oxide are used in the most severe CDH patients but are associated with a higher risk on surgical haemorrhage. The recurrence rate is associated with both the use of an abdominal patch and the presence of pulmonary hypertension, regardless of medical treatment.
\end{abstract}

Kim Heiwegen

Kim.Heiwegen@radboudumc.nl

1 Department of Pediatric Surgery, Radboudumc-Amalia Children's Hospital, Route 618, PO Box 9101, 6500 HB Nijmegen, The Netherlands

2 Department for Health Evidence, Radboud Institute for Health Sciences, Radboudumc, Nijmegen, The Netherlands

3 Department of Neonatology, Radboudumc-Amalia Children's Hospital, Nijmegen, The Netherlands

\section{Introduction}

Congenital diaphragmatic hernia $(\mathrm{CDH})$ is a potentially lethal birth defect caused by an improperly formed diaphragm during embryogenesis. Lung hypoplasia and pulmonary hypertension potentially cause severe respiratory failure which is challenging to treat in neonates. Different treatment interventions for $\mathrm{CDH}$ patients have been up for discussion for several years. One of those interventions is the use of extracorporeal membranous oxygenation (ECMO), which can be used in patients with respiratory failure and pulmonary hypertensive crisis resistant to gentle ventilation techniques [1]. ECMO oxygenates the 
blood outside the body, giving cardiopulmonary support by taking over gaseous exchange from the lungs [2]. Treatment with ECMO is associated with a higher survival rate in high-risk patients, although the overall survival of patients requiring ECMO has shown to be lower [3, 4]. Possible side effects of ECMO are the need for anticoagulation and, subsequently, the risk of haemorrhage. Despite the advances and higher survival in $\mathrm{CDH}$ patients, ECMO can be accompanied by a higher incidence of morbidity such as surgical complications, for which literature is scarce [5]. One of the important risk factors associated with surgical complications is the use of patches to close diaphragmatic defects tension-free [6,7]. Patch repair is associated with greater morbidity such as a higher recurrence rate of $\mathrm{CDH}$ than primary repair $[8,9]$. In the most severely affected neonates, both patch repair and use of ECMO are necessary, so they seem to have a higher risk of several surgical complications. For example, the development of a chylothorax is associated with both patch repair and treatment with ECMO without knowing the exact relationship between these factors [10]. To gain insight into the risk factors for the incidence of surgical complications, it is important to evaluate the contribution of different treatment options, such as ECMO and patch repair, but also $\mathrm{CDH}$ characteristics and clinical conditions, such as pulmonary hypertension. The aim of this study is to provide insight into possible related factors for developing surgical complications: haemorrhage, chylothorax, and recurrence of $\mathrm{CDH}$ within the first 12 months.

\section{Methods}

All neonates with CDH treated in the Radboudumc Amalia children's hospital Nijmegen, the Netherlands (European Reference Center), between 1 January 2000 and 1 January 2016, were included in this retrospective cohort study. The follow-up on these patients had to be at least 12 months for the surgical complications and mortality, based on recorded clinical and outpatient visits. Patients were managed according to the standard care for $\mathrm{CDH}$ patients at the time of attending, as described by the VICI trial and CDH registry [11]. ECMO was offered to patients with respiratory failure and pulmonary hypertension, where standard therapy and ventilation techniques had failed. The repair of the $\mathrm{CDH}$ was performed by experienced $\mathrm{CDH}$ pediatric surgeons, who aimed for a tension-free closure. A polytetrafluorethylene $\left(\right.$ GORE-TEX $\left.^{\circledR}\right)$ patch was used to construct a dome-shaped patch in all cases of patch repair. The hernia repair for patients during ECMO was targeted after stabilization and weaning, but before decannulation.

Primary outcomes of this study were surgical haemorrhages, chylothorax and recurrence of $\mathrm{CDH}$ up to the age of one. A surgical haemorrhage was scored as stated in the clinical and surgical records, such as peri-operative splenic bleedings or intraperitoneal haemorrhage. Chylothorax was diagnosed after drainage of chylus confirmed by laboratory determinations of lymphocytes and triglycerides. Recurrences were diagnosed on two-plain X-rays of the thorax, in which a significant bulging of the diaphragm or relocation of intra-abdominal organs into the thoracic cavity was considered a recurrence. A CT or MRI was only used where the X-ray was inconclusive.

Patient records were evaluated by two authors for outcome measures, demographics (such as gender and location of birth), and perinatal parameters, including Apgar score and birth weight. Defect size was documented according to the CDH Study Group (CDHSG) scale, in which A is a small defect ( $<25 \%$ of the hemidiaphragm), B is a defect including $25-50 \%$ of the hemidiaphragm, $\mathrm{C}$ is a defect of $50-75 \%$ of the hemidiaphragm, and in D, most of the hemidiaphragm is absent $(>75 \%)$ [12]. Pulmonary hypertension was scored if seen on ultrasonic scans or if appropriate medication was required (such as inhaled nitric oxide). After 2010 the criteria published by the $\mathrm{CDH}$ EURO consortium were used [13].

\section{Statistical analyses}

Parameters and outcomes are expressed as means with the standard deviations, median with the range, or in frequencies and percentages, where appropriate. For comparison of continuous variables or categorical data between two patient cohorts (such as ECMO vs. non-ECMO or primary vs. patch repair), the independent student T-test, Mann-Whitney $U$ test, Chi-square test, or Fisher exact tests were used where appropriate.

Multivariate logistic regression analyses were performed to evaluate the association of the hypothesized risk factors with the outcomes of surgical haemorrhage and chylothorax. The hypothesized potential risk factors were prenatal diagnosis of CHD (yes/no), side of CDH (left/ right/bilateral), liver-up and left-side hernia, because this is often described as severe (yes/no), pulmonary hypertension (yes/no), treatment with inhaled nitric oxide (iNO) (yes/ no), ECMO use (yes/no), repair with diaphragmatic patch (yes/no), and abdominal patch after $\mathrm{CDH}$ repair (yes/no). The odds ratios (OR) with $95 \%$ confidence intervals (CI) were first estimated for the separate potential risk factors, and thereafter an adjusted model was used including all potential risk factors. For the outcome recurrence of $\mathrm{CDH}$ within 1 year, a Cox regression analyses were used accounting for time to recurrence, calculating hazard ratios (HR) and 95\% CI in a crude and adjusted model for the risk factors. Only patients who were surgically repaired were included in these analyses. A p-value of $<0.05$ was 
considered statistically significant. All statistical analyses were performed using IBM SPSS Statistics Data Editor software program version 22 .

\section{Results}

\section{Study population and treatment}

Two hundred and fifteen patients were included in this study, of whom $58 \%$ were born in our centre. Eleven patients born in this period were excluded for several reasons such as diagnosis at a later age (three patients) or dying directly after birth (four patients). All included patients had a follow-up period of at least 1 year, which was the endpoint of follow-up of this study. They had a 1 -year survival rate of $73 \%$. After 1 year, ten patients were lost to follow-up for several reasons, but were contacted to ask whether they preferred follow-up by this centre or by contact with their peripheral centre [14]. ECMO was used in $31 \%$ of the patients $(n=66)$. All except one were treated with VA-ECMO. The mean ECMO run duration was 8.6 days (SD 3.6). Of the 66 ECMO patients, 38 patients did not survive up to the age of one. The mortality rate after 1 year was higher in patients requiring ECMO compared to those who did not $(58 \%$ vs. $14 \%, p<0.001)$. Of the 20 patients who did not survive and did not receive ECMO treatment, two patients had a chromosomal anomaly and five a cardiac anomaly. The diaphragm defects of fifteen of these patients were not repaired at all.

Of all 215 patients, the defects of 197 patients were surgically repaired. The approach to repair was performed by a subcostal incision in most patients (89\%), 3\% by thoracotomy while the remainder $(8 \%)$ was repaired in a minimally invasive fashion (four laparoscopic and twelve by thoracoscopy). Demographics of these 197 patients are displayed in Table 1, with specifics of the missing data presented below the table. Most patients were male (62\%) and more than half of the patients was prenatally diagnosed (54\%). Patch repair was required in $78(40 \%)$ of the patients. A more detailed description of surgical repair of these patients can be found in a previous overview article on this cohort [15].

Almost one third of the surgically repaired patients were cannulated on ECMO (63 patients). ECMO patients more often required a patch compared to the non-ECMO patients (49 patients $(64 \%)$ vs. 14 patients $(12 \%), p<0.001)$. Mortality after 1 year was significantly higher in patch repaired patients compared to primary repaired patients (34/78 vs. $6 / 119, p<0.001)$.
Table 1 Demographics and characteristics of the surgically repaired congenital diaphragmatic hernia patients

\begin{tabular}{|c|c|}
\hline & $\begin{array}{l}\text { Surgically repaired CDH } \\
\text { patients }(n=197)\end{array}$ \\
\hline Gender-male, $n(\%)$ & $122(61.9)$ \\
\hline Inborn $^{\mathrm{a}}, n(\%)$ & $106(56.1)$ \\
\hline Prenatal diagnosis ${ }^{\mathrm{b}}, n(\%)$ & $100(54.1)$ \\
\hline Birth weight, mean (SD) & $2968.9(678)$ \\
\hline \multicolumn{2}{|l|}{ Apgar scores, median (range) } \\
\hline $1 \mathrm{~min}$ & $7(0-9)$ \\
\hline $5 \min$ & $8(1-10)$ \\
\hline \multicolumn{2}{|l|}{ Side hernia ${ }^{\mathrm{c}}, n(\%)$} \\
\hline Left & $160(81.6)$ \\
\hline Right & $31(15.8)$ \\
\hline Bilateral & $5(2.6)$ \\
\hline \multicolumn{2}{|l|}{ Size hernia $^{\mathrm{d}}, n(\%)$} \\
\hline A & $16(15.4)$ \\
\hline B & $45(43.3)$ \\
\hline $\mathrm{C}$ & $36(34.6)$ \\
\hline $\mathrm{D}$ & $7(6.7)$ \\
\hline Liver up/left side ${ }^{\mathrm{e}}, n(\%)$ & $42(21.3)$ \\
\hline Pulmonary hypertension, $n(\%)$ & $89(45.2)$ \\
\hline Use of nitric oxide ${ }^{\mathrm{f}}, n(\%)$ & $77(40.3)$ \\
\hline $\mathrm{ECMO}, n(\%)$ & $63(32.1)$ \\
\hline \multicolumn{2}{|l|}{ Surgical repair } \\
\hline Type of repair: patch & 78 (39.6) \\
\hline Abdominal patch & $29(15.1)$ \\
\hline
\end{tabular}

ECMO extracorporeal membranous oxygenation

Missing values: ${ }^{\mathrm{a}} 8,{ }^{\mathrm{b}} 12,{ }^{\mathrm{c}} 1,{ }^{\mathrm{d}} 93,{ }^{\mathrm{e}} 5,{ }^{\mathrm{f}} 6$

\section{Surgical complications}

In total, 60 patients developed one of the three primary outcomes, such as a chylothorax, haemorrhage or recurrence within 1 year. Of these patients with complications, 23 patients (38\%) did not survive compared to 17 (12\%) of the patients without complications $(p<0.001)$. Patients with complications required surgery more often within the first year of life than those without complications (median 3 (range $1-12$ ) vs. 1 (range $1-11), p<0.001$ ).

\section{Surgical haemorrhage}

Twenty patients (10\%) developed a surgical haemorrhage, ten had a splenic laceration, three developed a haemothorax, while the remaining patients had sanguineous abdominal leakage based on liver laceration and wound surface haemorrhage. Twelve of these patients $(60 \%) \mathrm{did}$ not survive, compared to $16 \%$ of the surgically repaired patients without surgical haemorrhage $(p<0.001)$. Eighteen of the twenty patients were cannulated on ECMO 
Table 2 The association between several treatment and clinical characteristics on the outcome of haemorrhage in a group of surgically repaired patients with congenital diaphragmatic hernia

\begin{tabular}{|c|c|c|c|c|}
\hline & Yes $(n=20) N(\%)$ & No $(n=177) N(\%)$ & $\mathrm{OR}_{\text {crude }}(95 \% \mathrm{CI})$ & $\mathrm{OR}_{\text {adjusted }}(95 \% \mathrm{CI})^{*}$ \\
\hline Chylothorax & $4(20.0)$ & $29(16.4)$ & $1.3(0.4-4.1)$ & $0.4(0.1-1.8)$ \\
\hline Patch for abdominal defect ${ }^{\mathrm{a}}$ & $9(45.0)$ & $19(11.0)$ & $6.6(2.4-17.9)$ & $1.2(0.3-4.7)$ \\
\hline ECMO used $^{\mathrm{b}}$ & $18(90.0)$ & $45(25.6)$ & $26.2(5.8-117.4)$ & $10.5(1.3-87.1)$ \\
\hline Type of surgical repair-patch & $15(75.0)$ & $63(35.6)$ & $5.4(1.9-15.6)$ & $2.3(0.5-10.5)$ \\
\hline Prenatal diagnosis of $\mathrm{CDH}^{\mathrm{c}}$ & $12(60.0)$ & $87(52.7)$ & $1.3(0.5-3.5)$ & $0.8(0.2-2.9)$ \\
\hline Liver-up and left-side hernia ${ }^{d}$ & $6(30.0)$ & $36(21.1)$ & $1.6(0.6-4.5)$ & $0.3(0.1-1.8)$ \\
\hline Pulmonary hypertension ${ }^{\mathrm{e}}$ & $13(81.3)$ & $73(46.8)$ & $4.9(1.4-18.0)$ & $0.2(0.02-1.9)$ \\
\hline Nitric oxide treatment ${ }^{\mathrm{d}}$ & $18(90.0)$ & $59(34.5)$ & $17.1(3.8-76.1)$ & $13.0(1.1-159.0)$ \\
\hline
\end{tabular}

$C D H$ congenital diaphragmatic hernia, ECMO extracorporeal membranous oxygenation

*Adjusted for all other factors. Bold values are the ones that are statistical significant

Missing values: ${ }^{\mathrm{a}} 5,{ }^{\mathrm{b}} 1,{ }^{\mathrm{c}} 12,{ }^{\mathrm{d}} 6,{ }^{\mathrm{e}} 25$

during diaphragmatic hernia repair. The patients requiring ECMO had a higher chance of developing surgical haemorrhage compared to the patients who did not (29\% vs. $2 \%$, $p<0.001)$. The incidence was highest in the group who required both ECMO and patch repair (14/49), when compared with patients with no ECMO and primary repair (2/105), resulting in an OR of 20.6 (95\% CI 4.5-95.2). The logistic regression analyses (Table 2) showed that multiple possible risk factors were significantly associated with haemorrhage in the crude models. However, after adjustment for each other, only treatment with ECMO and iNO remained significant, with, respectively, a 10 and 13 times higher chance of developing surgical haemorrhage.

\section{Chylothorax}

A chylothorax was diagnosed in 33 patients, who all required treatment. A medium chain triglyceride (MCT) diet was used in 27 patients and/or thorax drainage in 32 patients. There were more patients with chylothorax that did not survive compared to patients without chylothorax (11 $(33 \%)$ vs. $17(18 \%), p=0.04)$. In the group of surgically repaired patients cannulated on ECMO, $20(32 \%)$ developed a chylothorax compared to thirteen (10\%) of the non-ECMO patients $(p<0.001)$. Patients who were repaired with a patch had a higher rate of chylothorax than those who were primary repaired (22/78 vs. 11/119, $p<0.001)$. The group of patients who required both ECMO and patch repair had the highest chance of developing a chylothorax (17/49).

The crude ORs of almost all potential risk factors were significantly associated with chylothorax, except for surgical haemorrhage and liver-up/left-side hernia (Table 3). However, when these associations were adjusted for each other, none remained significantly associated to chylothorax, which was also the case for ECMO treatment (OR (95\% CI):1.6 (0.5-5.2)) and patch repair (OR $(95 \%$ CI):2.1 (0.7-6.1)) independently.

\section{Recurrence}

Twelve patients $(6 \%)$ suffered a recurrence within 1 year of follow-up. Seven (58\%) of these twelve patients were treated with ECMO while five $(42 \%)$ were not $(p=0.06)$. The recurrence rate was not significantly different in patch versus primary repaired patients (5/78 vs. $7 / 119$ (both $6 \%$ ), $p=1.00)$. Table 4 shows the Cox regression analysis of this outcome measure. Two patients were excluded due to unknown date of death. The crude HRs for abdominal patch, ECMO, pulmonary hypertension and iNO were significantly associated with recurrence. After adjustment for each other, only abdominal patch (HR (95\% CI):11.3 (1.5-84.4)) and pulmonary hypertension (HR $(95 \%$ CI):10.0 (1.5-65.8)) remained independently associated with recurrence. Two of the five patients with recurrence and abdominal patch required a second patch before definite closure (of the total of five patients who required a second abdominal patch in this cohort). In two patients the patch could be removed after 38 days and 55 days, respectively, without any complications, while the fifth patient had severe bowel necrosis and even required a final ileostomy.

\section{Discussion}

Patients with $\mathrm{CDH}$ are often difficult to treat and caregivers face challenges and unexpected events every day. The closure of the diaphragmatic defects remains a surgical challenge. This study evaluates the association of several 
Table 3 The association between several treatment and clinical characteristics on the outcome of chylothorax in a group of surgically repaired patients with congenital diaphragmatic hernia

\begin{tabular}{|c|c|c|c|c|}
\hline & Yes $(n=33) N(\%)$ & No $(n=164) N(\%)$ & $\mathrm{OR}_{\text {crude }}(95 \% \mathrm{CI})$ & $\mathrm{OR}_{\text {adjusted }}(95 \% \mathrm{CI})^{*}$ \\
\hline Surgical haemorrhage & $4(12.1)$ & $16(9.8)$ & $1.3(0.4-4.1)$ & $0.3(0.1-1.4)$ \\
\hline Patch for abdominal defect ${ }^{\mathrm{a}}$ & $9(27.3)$ & 19 (11.9) & $2.8(1.1-6.8)$ & $0.9(0.3-3.1)$ \\
\hline ECMO used ${ }^{\mathrm{b}}$ & 20 (60.6) & $43(26.4)$ & $4.3(2.0-9.4)$ & $1.6(0.5-5.2)$ \\
\hline Type of surgical repair-patch & $22(66.7)$ & $56(34.1)$ & $3.8(1.7-8.5)$ & $2.1(0.7-6.1)$ \\
\hline Prenatal diagnosis of $\mathrm{CDH}^{\mathrm{c}}$ & $23(71.9)$ & 76 (49.7) & $2.6(1.1-6.0)$ & $1.7(0.6-4.9)$ \\
\hline Liver-up and left-side hernia ${ }^{d}$ & $11(34.4)$ & $31(19.5)$ & $2.2(0.9-5.0)$ & $1.1(0.4-3.5)$ \\
\hline Pulmonary hypertension ${ }^{\mathrm{e}}$ & $25(80.6)$ & $61(43.3)$ & $5.5(2.1-14.1)$ & $2.2(0.5-10.1)$ \\
\hline Nitric oxide treatment ${ }^{\mathrm{d}}$ & $24(72.7)$ & $53(33.5)$ & $5.3(2.3-12.2)$ & $3.1(0.8-12.4)$ \\
\hline
\end{tabular}

$C D H$ congenital diaphragmatic hernia, ECMO extracorporeal membranous oxygenation

Missing values: ${ }^{\mathrm{a}} 5,{ }^{\mathrm{b}} 1,{ }^{\mathrm{c}} 12,{ }^{\mathrm{d}} 6,{ }^{\mathrm{e}} 25$

*Adjusted for all other factors. Bold values are the ones that are statistical significant

Table 4 The association between several treatment and clinical characteristics on the outcome of recurrence within the age of 1 year in a group of surgically repaired patients with congenital diaphragmatic hernia

\begin{tabular}{llllr}
\hline & Yes $(n=12) N(\%)$ & No $(n=183) N(\%)$ & HR $_{\text {crude }}(95 \%$ CI $)$ & HR adjusted $(95 \% \text { CI })^{*}$ \\
\hline Surgical haemorrhage & $2(16.7)$ & $18(9.8)$ & $4.0(0.9-18.7)$ & $3.3(0.3-37.8)$ \\
Chylothorax & $3(25.0)$ & $29(15.8)$ & $2.1(0.6-7.7)$ & $1.0(0.2-4.3)$ \\
Patch for abdominal defect $^{\mathrm{a}}$ & $5(41.7)$ & $23(12.9)$ & $\mathbf{8 . 2}(\mathbf{2 . 6}-\mathbf{- 2 5 . 9})$ & $\mathbf{1 1 . 3}(\mathbf{1 . 5}-\mathbf{8 4 . 4})$ \\
ECMO used $^{\mathrm{b}}$ & $7(58.3)$ & $55(30.2)$ & $\mathbf{6 . 3}(\mathbf{2 . 0 - 2 0 . 1})$ & $0.6(0.1-5.7)$ \\
Type of surgical repair-patch & $5(41.7)$ & $72(39.3)$ & $1.8(0.6-5.5)$ & $0.8(0.2-3.7)$ \\
Prenatal diagnosis of CDH $^{\mathrm{c}}$ & $6(50.0)$ & $93(54.4)$ & $1.0(0.3-3.2)$ & $0.4(0.1-2.1)$ \\
Liver-up and left-side hernia $^{\mathrm{d}}$ & $4(33.3)$ & $38(21.5)$ & $2.1(0.6-7.1)$ & $2.9(0.5-17.1)$ \\
Pulmonary hypertension $^{\mathrm{e}}$ & $9(81.8)$ & $76(47.5)$ & $\mathbf{7 . 3}(\mathbf{1 . 6}-\mathbf{3 3 . 8})$ & $\mathbf{1 0 . 0}(\mathbf{1 . 5}-\mathbf{6 5 . 8})$ \\
Nitric oxide treatment $^{\mathrm{d}}$ & $7(58.3)$ & $68(38.4)$ & $\mathbf{3 . 4}(\mathbf{1 . 1}-\mathbf{1 0 . 9})$ & $0.4(0.1-2.0)$ \\
\hline
\end{tabular}

$C D H$ congenital diaphragmatic hernia, ECMO extracorporeal membranous oxygenation, $H R$ hazard ratio

Missing values: ${ }^{\mathrm{a}} 5,{ }^{\mathrm{b}} 1,{ }^{\mathrm{c}} 12,{ }^{\mathrm{d}} 6,{ }^{\mathrm{e}} 24$

*Adjusted for all other factors. Bold values are the ones that are statistical significant

hypothesized risk factors, such as ECMO treatment and patch repair, on the development of surgical complications. After a multivariate logistic regression analysis was performed, a few surprising associations were found such as the association of inhaled nitric oxide with haemorrhage and the initial need for an abdominal patch and pulmonary hypertension with the development of a recurrence of the diaphragmatic hernia within 1 year of follow-up.

\section{Surgical haemorrhage}

As expected, our data show that ECMO is associated with the development of surgical haemorrhage. This can partly be explained by the fact that these patients are treated with anticoagulation medication (for example, heparin), which makes these neonates more vulnerable to bleeding complications [16]. Patients who required both ECMO and a patch had the highest chance of developing surgical haemorrhage. However, after adjusting for other risk factors, patch repair on its own did not seem to be associated with this outcome. Remarkably, inhaled nitric oxide seemed to raise the chance of haemorrhage, while pulmonary hypertension itself did not. Nitric oxide is a vasodilator and is used in cases of severe respiratory failure. However, side effects are decreased platelet aggregation, surfactant dysfunction and an increased risk of bleeding [17]. There has been a lot of discussion on the role of iNO treatment in CDH patients and its use is highly variable among centres all over the world [18]. Some suggest that iNO does not influence outcomes such as bleeding complications [19]. However, a multicentre study (2016) of the CDH registry stated that iNO use worldwide 
is highly variable and does not seem to be beneficial and may even be associated with an increased mortality rate [18]. Our study adds evidence that it may raise the rate of surgical haemorrhage and the indications in favour of using it during surgery should be well thought out.

\section{Chylothorax}

It has been previously described that ECMO or patch repair in $\mathrm{CDH}$ patients both increase the incidence of chylothorax [20, 21]. In this study, patients with both ECMO and patch repair had the highest chance of developing this complication, but independently they did not increase the risk for chylothorax. There are different hypotheses on why $\mathrm{CDH}$ patients develop a chylothorax such as rupture of the lymph vessels peri-operatively during $\mathrm{CDH}$ repair or due to a high pressure of abdominal organs intra-thoracically (so more damage to lymph vessels), which could also explain the relationship with patch repair, in which the large defect enables more organs to protrude into the thoracic cavity [22]. The association between ECMO and chylothorax has also been described, and it was hypothesized that the pressure in the ductus thoracicus becomes too high causing chylus [22]. Previously, timing of repair was also associated with the odds of developing chylothorax, as only the patients repaired before ECMO did not show an increased risk on chylothorax [21]. None of the patients in this cohort were repaired before ECMO cannulation, but still no correlation could be found.

\section{Recurrence}

Recurrence in this group was not significantly associated with ECMO treatment or patch repair. Moreover, the combination of these two treatments did not seem to influence the incidence of short term recurrences. There was no significant difference in primary versus patch repaired patients, which could be due to technical factors such as the manner of patch implementation and avoidance of tension on sutures, which seem to have an influence on developing a recurrence [7, 23]. In our analysis, an abdominal patch, however, seemed to be associated with recurrences (OR 95\% CI 11.3 (1.5-84.4)), because almost half of patients with recurrences $(42 \%)$ initially required an abdominal patch. Delayed abdominal closure after initial requirement of an abdominal patch is more likely when patients are repaired while cannulated on ECMO [24]. The use of abdominal patches has been described as a predictor of mortality, and these patients are more likely to require a higher amount of blood transfusions while on ECMO $[24,25]$. In our cohort, mortality rate after 1 year was high as well $(57 \%)$ where an abdominal patch was needed. However, a clear association with development of recurrences has not yet been described before. One of the causes could be the severity of the disease in these neonates, because an abdominal patch is used mainly in patients with larger defects; however, half of the defect sizes were unknown in the patients with recurrence. Another cause could be the closure of the abdomen in patients with large defects, which gives an increased intraabdominal volume but may still give a relatively higher intra-abdominal pressure as well during and after surgery. This could lead to a recurrence of the defect due to the high pressure on the diaphragm. Another predictor for recurrence in our study was the presence of pulmonary hypertension during initial hospitalization. This could be due to the underlying pathology, or due to the treatment of the pulmonary hypertension, although we did not find an independent association with the use of iNO. Moreover, there is only a small number of recurrences in this cohort which could possibly be of influence on the significance of these risk factors. A longer follow-up in a larger cohort and prospective data gathering could give more insight into the causes of the recurrence in children with pulmonary hypertension. However, a previous study on the long term outcomes of this research group did not show significantly more recurrences (three after the age of 1 year of age) in the longer follow-up, although ten patients did not complete the long-term follow-up [14].

As this study shows, several factors are associated with different types of surgical complications. Due to its retrospective nature, this data collection may be less reliable. Therefore, our findings will need to be confirmed prospectively. However, the incidence of surgical complications is influenced by multiple factors and these factors could influence surgical timing and peri-operative management to improve surgical outcome. Further steps for possible prevention of these complications are required and need to be prospectively evaluated.

\section{Conclusion}

The rate of surgical complications in congenital diaphragmatic hernia patients increases with their increasing survival. Bleeding complications are associated with the use of ECMO treatment and inhaled nitric oxide. Furthermore, the incidence of a recurrence within 1 year seems to be associated with use of an abdominal patch and presence of pulmonary hypertension, regardless of treatment given.

\section{Compliance with ethical standards}

Conflict of interest All authors have no conflict of interest. 
Ethical approval This study was approved by the local medical ethical committee, and all data was processed anonymously.

Open Access This article is licensed under a Creative Commons Attribution 4.0 International License, which permits use, sharing, adaptation, distribution and reproduction in any medium or format, as long as you give appropriate credit to the original author(s) and the source, provide a link to the Creative Commons licence, and indicate if changes were made. The images or other third party material in this article are included in the article's Creative Commons licence, unless indicated otherwise in a credit line to the material. If material is not included in the article's Creative Commons licence and your intended use is not permitted by statutory regulation or exceeds the permitted use, you will need to obtain permission directly from the copyright holder. To view a copy of this licence, visit http://creativecommons. org/licenses/by/4.0/.

\section{References}

1. Wilkinson DJ, Losty PD (2013) Management of congenital diaphragmatic hernia. Paediatr Child Health 24:23-26

2. Mugford M, Elbourne D, Field D (2008) Extracorporeal membrane oxygenation for severe respiratory failure in newborn infants. Cochrane Database Syst Rev 16:CD001340

3. Morini F, Lally PA, Lally KP et al (2015) The congenital diaphragmatic hernia study group registry. Eur J Pediat Surg Off J Austrian Assoc Pediat Surg 25:488-496

4. Morini F, Goldman A, Pierro A (2006) Extracorporeal membrane oxygenation in infants with congenital diaphragmatic hernia: a systematic review of the evidence. Eur J Pediatr Surg Off $\mathbf{J}$ Austrian Assoc Pediatr Surg 16:385-391

5. Yokota K, Uchida H, Kaneko K et al (2014) Surgical complications, especially gastroesophageal reflux disease, intestinal adhesion obstruction, and diaphragmatic hernia recurrence, are major sequelae in survivors of congenital diaphragmatic hernia. Pediatr Surg Int 30:895-899

6. Losty PD (2014) Congenital diaphragmatic hernia: where and what is the evidence? Semin Pediatr Surg 23:278-282

7. Puligandla PS, Grabowski J, Austin M et al (2015) Management of congenital diaphragmatic hernia: a systematic review from the APSA outcomes and evidence based practice committee. J Pediatr Surg 50:1958-1970

8. Danzer E, Hedrick HL (2014) Controversies in the management of severe congenital diaphragmatic hernia. In: Seminars in fetal \& neonatal medicine, vol 19, pp 376-384

9. Laituri CA, Garey CL, Valusek PA et al (2010) Outcome of congenital diaphragmatic hernia repair depending on patch type. Eur J Pediatr Surg Off J Austrian Assoc Pediatr Surg 20:363-365

10. Costa KM, Saxena AK (2018) Surgical chylothorax in neonates: management and outcomes. World J Pediatr 14:110-115

11. van den Hout L, Tibboel D, Vijfhuize S et al (2011) The VICItrial: high frequency oscillation versus conventional mechanical ventilation in newborns with congenital diaphragmatic hernia: an international multicentre randomized controlled trial. BMC Pediatr 11:98

12. Lally KP, Lasky RE, Lally PA et al (2013) Standardized reporting for congenital diaphragmatic hernia - an international consensus. J Pediatr Surg 48:2408-2415

13. Reiss I, Schaible T, van den Hout L et al (2010) Standardized postnatal management of infants with congenital diaphragmatic hernia in Europe: the CDH EURO Consortium consensus. Neonatology 98:354-364

14. Janssen S, Heiwegen K, van Rooij IA et al (2018) Factors related to long-term surgical morbidity in congenital diaphragmatic hernia survivors. J Pediatr Surg 53:508-512

15. Heiwegen K, de Blaauw I, Janssen S et al (2017) Short term surgical outcomes in the treatment of congenital diaphragmatic hernia: an overview of 15 years experience. Ann Emerg Surg 2:1005

16. Sy E, Sklar MC, Lequier L et al (2017) Anticoagulation practices and the prevalence of major bleeding, thromboembolic events, and mortality in venoarterial extracorporeal membrane oxygenation: a systematic review and meta-analysis. J Crit Care 39:87-96

17. Peliowski A, Canadian Paediatric Society, Fetus and Newborn Committee (2012) Inhaled nitric oxide use in newborns. Paediatr Child Health 17:95-100

18. Putnam LR, Tsao K, Morini F et al (2016) Evaluation of variability in inhaled nitric oxide use and pulmonary hypertension in patients with congenital diaphragmatic hernia. JAMA Pediatr 170:1188-1194

19. Yang Y, Feng Y, Zhou XG et al (2016) Inhaled nitric oxide in preterm infants: an updated meta-analysis. J Res Med Sci Off J Isfahan Univ Med Sci 21:41

20. Gonzalez R, Bryner BS, Teitelbaum DH et al (2009) Chylothorax after congenital diaphragmatic hernia repair. J Pediatr Surg 44:1181-1185

21. Levy SM, Lally PA, Lally KP et al (2013) The impact of chylothorax on neonates with repaired congenital diaphragmatic hernia. J Pediatr Surg 48:724-729

22. Zavala A, Campos JM, Riutort C et al (2010) Chylothorax in congenital diaphragmatic hernia. Pediatr Surg Int 26:919-922

23. Tsai J, Sulkowski J, Adzick NS et al (2012) Patch repair for congenital diaphragmatic hernia: is it really a problem? J Pediatr Surg 47:637-641

24. Laje P, Hedrick HL, Flake AW et al (2016) Delayed abdominal closure after congenital diaphragmatic hernia repair. J Pediatr Surg 51:240-243

25. Jawaid WB, Qasem E, Jones MO et al (2013) Outcomes following prosthetic patch repair in newborns with congenital diaphragmatic hernia. Br J Surg 100:1833-1837

Publisher's Note Springer Nature remains neutral with regard to jurisdictional claims in published maps and institutional affiliations. 